\title{
Stroke mortality audit using the Structured Judgement Review method
}

\author{
Authors: Jade Thomas, ${ }^{A}$ Kyaw Lin Saw ${ }^{B}$ and Katja Adie ${ }^{C}$
}

Mortality data provided by the Sentinel Stroke National Audit Programme demonstrated the Royal Cornwall Hospitals Trust (RCHT) to have a higher than national average mortality ratio. ${ }^{1}$ In response to this, the RCHT stroke department undertook a mortality review of patients admitted with stroke making use of the Structured Judgement Review (SJR) process. $^{2}$

The review found all patients were deemed as receiving adequate, good or excellent care. There were no cases where death was deemed as definitely avoidable. The team found the SJR to be a useful, validated tool for mortality review though recognised specific limitations to its use and wider limitations within our review process. Focused areas for improvement derived from the review included improving compliance with local palliative care guides, improved documentation, links with primary care via Care Quality Commission atrial fibrillation group and consideration of improved scanning facilities. We also acknowledged wider unaccounted factors which may impact stroke mortality and thus influence perceived mortality ratios.

KEYWORDS: Stroke, mortality, structured judgement review, mortality review, stroke care

\section{Introduction}

The Sentinel Stroke National Audit Programme (SSNAP) captures performance data on stroke care across acute trusts in England and Wales. Audit data encompasses all aspects of stroke care including thrombolysis, complication rates and overall mortality. SSNAP gives a standardised mortality ratio (SMR) allowing comparison of mortality rates between trusts. The April 2015-March 2016 mortality ratio for the Royal Cornwall Hospitals Trust (RCHT) was higher than national average, showing the RCHT to be close to being an outlier for stroke-associated mortality (SMR 1.24). ${ }^{1}$

In response to this, the RCHT stroke department undertook a mortality review of patients admitted with stroke. The review made use of the Structured Judgement Review (SJR) process introduced by the Royal College of Physicians (RCP). ${ }^{2}$ This report discusses the mortality review methodology, its results, and local implications for stroke care.

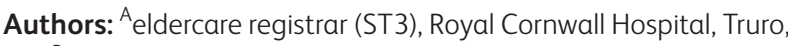
UK; ${ }^{B}$ trust doctor (stroke medicine,) Royal Cornwall Hospital, Truro, UK; C Consultant geriatrician, Royal Cornwall Hospital, Truro, UK

\section{Methods}

Patients admitted with stroke between 1 April 2016 and 31 March 2017 who died within 30 days were included within the review. The Hospital Episode Statistics (HES) system was used to identify patients using clinical diagnostics coding (G46, I60, I61, I62, I63, I64, I66). The hospital mortality committee requested 50 patients selected at random by the data analysis team for inclusion within the review.

Demographic data collected included gender, age and stroke type. Time to consultant review was also incorporated within data collection.

Case notes were analysed using the SJR developed as part of the RCP 2016 National Mortality Case Record Review Programme. ${ }^{2,3}$ The SJR is a two stage review process which has been validated and used in practice within a large NHS region. ${ }^{2}$ The review requires an assessor to provide judgement scores on care provided at different phases of the patient journey. Phases of patient care include: admission and initial care, perioperative and procedural care, ongoing care, and end-of-life care (or discharge care.) The 'front-line' reviewers analyse the cases and score the level of care accordingly from 1 (poor) to 5 (excellent). The reviewers are encouraged to use explicit qualitative statements, such as 'failure of / unsatisfactory / best practice / inadequate in'. Such statements provide evidence for the score allocated. The final part of the SJR looks at avoidability of death (see Table 1). This subjective rating accounts for all information from the case note review. It is scored from 1 (definitely avoidable) to 6 (definitely not avoidable). If the initial reviewer score was 1 or 2 (very poor or poor accordingly,) then a second review takes place within the hospital governance process. It provides a validation process for the first reviewer's concern.

The SJR for our review was undertaken by stroke consultants and registrars working on the stroke unit.

Table 1. Avoidability of death score

$\begin{array}{ll}\text { Score } & \text { Description } \\ 1 & \text { Definitely avoidable } \\ 2 & \text { Strong evidence of avoidability } \\ 3 & \text { Probably avoidable (more than } 50: 50 \text { ) } \\ 4 & \text { Possibly avoidable but not very likely (less than 50:50) } \\ 5 & \text { Slight evidence of avoidability } \\ 6 & \text { Definitely not avoidable }\end{array}$




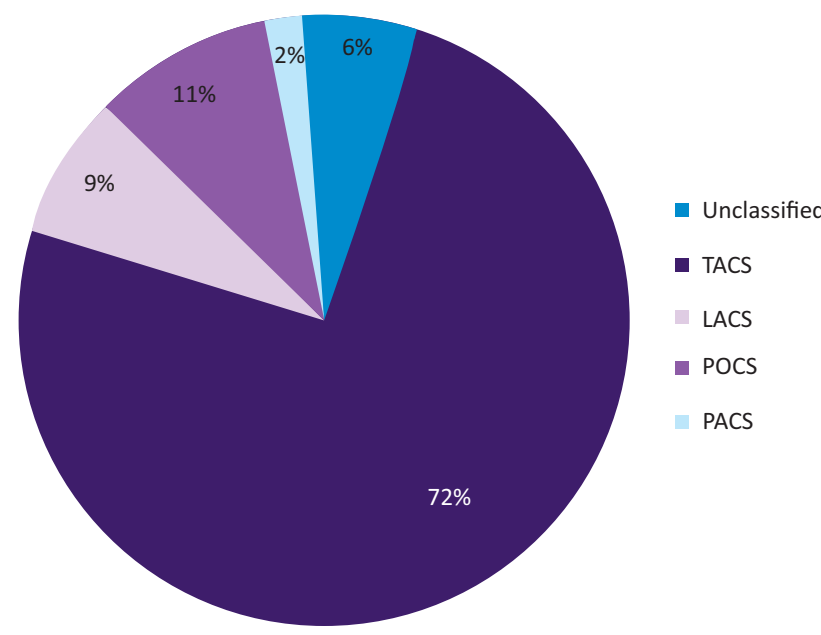

Fig 1. Oxford Stroke Classification. TACS = total anterior circulation stroke, LACS = lacunar syndrome, POCS = posterior circulation syndrome, $\mathrm{PACS}=$ partial anterior circulation syndrome.

\section{Results}

A total of 1,027 patients were admitted to the RCHT with a stroke and of these, 201 patients died (19\%). The expected number of deaths was $162(15 \%)$, giving a standardised mortality ratio of 1.24 . Of the 50 patients that were reviewed, $3(6 \%)$ were found to have a different diagnosis than stroke (traumatic intracerebral bleed $n=2$, subarachnoid haemorrhage $n=1$ ) and were subsequently excluded from the review. Of the remaining 47 cases, $55 \%$ were female and $45 \%$ male with average age of 82 years. There was a largely equal number of ischaemic to haemorrhagic strokes seen, 25 (54\%) vs 22 (46\%) respectively. Fig 1 shows the proportion of patients by Oxford Stroke Classification, demonstrating the majority to have suffered a total anterior circulation event.

Table 2 demonstrates results for SJR phases of care scores. Overall, clinical care was deemed to be good or excellent with scores of 3 (adequate care) or above being seen for all patients. At the initial management phase, $83 \%$ of the cases were reviewed as excellent care (care score 5). Similarly, $82 \%$ were deemed excellent for the ongoing care phase, and $75 \%$ for end-of-life care. Overall care scores showed $72 \%$ as having a rating of excellent.

\section{Table 2. Phases of care}

\begin{tabular}{lllll} 
Care score & $\begin{array}{l}\text { Initial } \\
\text { management }\end{array}$ & $\begin{array}{l}\text { Ongoing } \\
\text { care }\end{array}$ & $\begin{array}{l}\text { EOL } \\
\text { care }\end{array}$ & $\begin{array}{l}\text { Overall } \\
\text { care }\end{array}$ \\
5 (excellent) & 39 & 37 & 33 & 34 \\
4 & 5 & 8 & 8 & 10 \\
3 & 3 & 0 & 3 & 3 \\
2 & 0 & 0 & 0 & 0 \\
1 (poor) & 0 & 0 & 0 & 0 \\
Not scored & 0 & 2 & 3 & 0 \\
Overall & 47 & 45 & 44 & 47 \\
\hline EOL = end-of-life & & & &
\end{tabular}

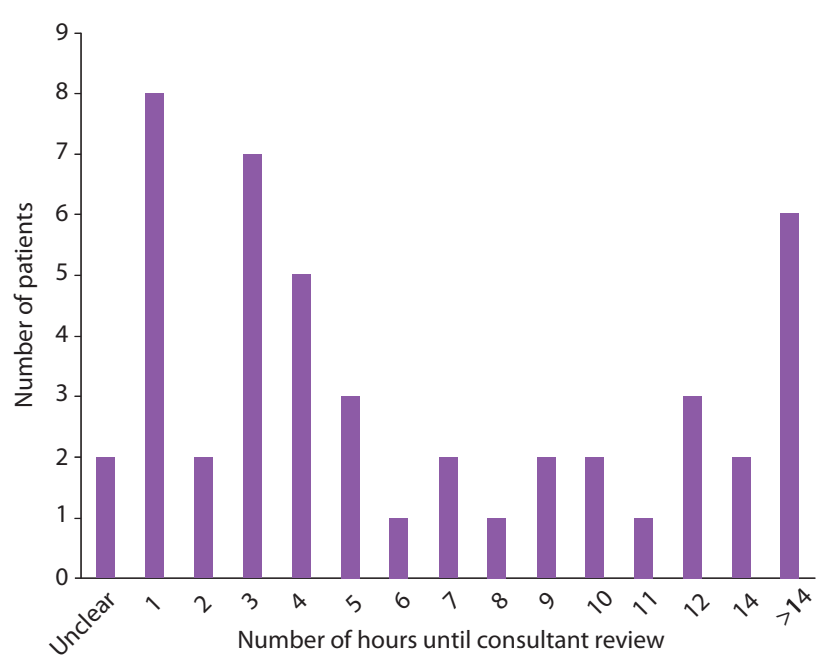

Fig 2. Time to consultant review.

Qualitative comments included early consultant review, prompt family discussions, and good interspecialty working. Fig 2 shows that the majority of patients were reviewed by a stroke consultant within 14 hours of admission ( $88 \%$ ).

Rationale given for a care score of 3 (adequate care) included delay in thrombolysis due to having to repeat a computed tomography (CT) brain scan as the first one was of poor quality, delay in checking of blood results for thrombolysed patient and poor documentation of discussion with family in patient notes.

For the end-of-life care phase a further three cases were deemed adequate for the following reasons: poor documentation of transfer, no documentation of certified cause of death in notes and non-compliance with trust palliative protocols.

Within the judgement for avoidability of death there were three cases in which a score of 5 (some evidence of avoidability) was given. The reasoning provided for this score included the above comments and an additional comment for a patient who sustained an ischaemic stroke, had known atrial fibrillation (AF), was not anticoagulated in the community and there were no obvious contraindications.

\section{Discussion}

From the structured review all patients were deemed as receiving adequate, good or excellent care. There were no cases where death was deemed as definitely avoidable. Areas flagged for improvement included management of AF, upgrading the CT scanner, improved documentation of death and consistent use of palliative care guidance. The results of the review have been presented to the executive team and shared within the stroke working group.

There are several issues that may have impacted our mortality rate. Firstly three out of 50 patients $(6 \%)$ were wrongly coded and this may have had an impact on the overall number of patients coded as stroke and increased mortality figures. This issue has been addressed with the coding team and a monthly meeting with coders, stroke data collectors and clinicians, instigated to harmonise coding. 
Secondly, the review flagged a patient with known AF who was not on anticoagulation treatment prior to stroke. This is not an isolated occurrence and we know that AF detection and anticoagulation treatment reduces stroke. ${ }^{4}$ This issue has been raised with the primary care team leads, community pharmacy leads and commissioners. Several initiatives are being explored in the community to improve AF detection and management, including the use of handheld ECG monitors and blood pressure devices.

Thirdly, a significant proportion of our patients had large ischaemic strokes that carry a poor prognosis. The standardised mortality ratio calculation provided by SSNAP has been validated as a tool, ${ }^{5}$ it takes stroke severity into account by including the National Institutes of Health Stroke Scale. Other factors included are known AF. Additional comorbidities, social deprivation and patient wishes with regards to escalation of care / resuscitation are however not taken into account. Our population of stroke patients is older than the national average with expected higher comorbidity and frailty rates which may have an impact on stroke mortality.

With respect to the SJR, assessors found it to be a useful tool for mortality review. Scoring of care phases allows easy overview and comparison with future mortality reviews. However, the review takes time to complete (15-45 min/case) so it produces a substantial workload depending on the number of patients reviewed.

There are several limitations to our review. The most important limitation is that SJR is a generic tool and does not allow disease specific review. It therefore did not take into account other important factors that influence stroke mortality such as timely admission to the stroke unit. ${ }^{6}$ Our stroke unit was in the overall category D of the SSNAP indicating poor performance with respect to acute stroke indicators. For instance only $37 \%$ of patients were admitted within 4 hours to the acute stroke unit (the national average is $59 \%){ }^{7}$ This will have had an effect on mortality.

Our review was limited to a random selection of patients and did not include all patients who died of stroke in the time period, and we may have missed other significant issues. Unfortunately resource constraints did not allow review of all patients.

A final limitation was that the review was conducted by consultants and registrars within the stroke department raising opportunity for judgement bias. Mortality review is also prone to reviewer bias, leading reviewers to judge that a case has received a lower quality of care than it actually has. ${ }^{2}$ An interdepartmental review is recommended within the SJR guidance as sufficient specialist knowledge of impact of care on outcomes is required, alongside an in-depth knowledge of the local policies and patient pathway in order to make department-specific recommendations. ${ }^{2,3,7}$ These issues were raised by the reviewers with the trust's mortality committee prior to audit but there was no resource for another department to complete the review.

\section{Conclusion}

The SJR provides a structured approach to mortality review that is reproducible and provides data for ongoing comparisons. Its use within our department has helped to focus on areas that require qualitative improvement. Specific areas for change include improving compliance with local palliative care guides, improved documentation, links with primary care via the Care Quality Commission AF group and consideration of improved scanning facilities. We hope that implementation of these recommendations in combination with working on improvement of SSNAP acute stroke indicators of good care (eg timely admission to stroke unit) will improve patient care and reduce stroke mortality.

\section{Acknowledgements}

We would like to thank Mr Richard Barrett, cardiac systems administrator, for support with data ascertainment.

\section{References}

1 Sentinel Stroke National Audit Programme. SSNAP key indicators. CCG portfolio April 2015-March 2016. London: King's College London, 2016. www.strokeaudit.org/results/Clinical-audit/ClinicalCCG-LHB-LCG.aspx [Accessed 9 May 2018].

2 Hutchinson A, Coster JE, Cooper KL et al. A structured judgement method to enhance mortality case note review: development and evaluation. BMJ Quality and Safety 2013;22:1032-40.

3 Royal College of Physicians. Using the structured judgement review method - a clinical governance guide to mortality case record reviews. London: RCP, 2016. www.rcplondon.ac.uk/sites/default/files/ media/Documents/NMCRR \% 20clinical \% 20governance \% 20guide_1. pdf?token=AS-qWBcA [Accessed 9 May 2018].

4 Hart RG, Pearce LA, Aguilar MI. Meta-analysis: Antithrombotic therapy to prevent stroke in patients who have nonvalvular atrial fibrillation. Ann Intern Med 2007;146:857-67.

5 Bray BD, Campbell J, Cloud GC et al. Derivation and external validation of a case mix model for the standardized reporting of 30-day stroke mortality rates. Stroke 2014:45:3374-80.

6 Dennis MS, Burn JPS, Sandercock PAG et al. Long-term survival after first-ever stroke: the Oxfordshire Community Stroke Project. Stroke 1993;24:796-800.

7 National Quality Board. National guidance on learning from deaths. London: NHS England, 2017. www.england.nhs.uk/publication/ national-guidance-on-learning-from-deaths/ [Accessed July 2017].

Address for correspondence: Dr Katja Adie, Care of Elderly Department, Royal Cornwall Hospital Trust, Truro, Cornwall TR1 3LJ, UK.

Email:k.adie@nhs.net 